\title{
Research on Forming Spring back of Cushion Segment for Automobile Clutch
}

\author{
Xiaoli Wang ${ }^{1, a}$, Rui Mu, b and Caihong Wang ${ }^{2, c}$ \\ ${ }^{1}$ Lianyungang Technical College, Lianyungang 222006, China; \\ ${ }^{2} Z$ hejiang King Kong Automobile Limited Company, Taizhou 318000, China. \\ ajslygxpwxl@126.com, bjslygmr@126.com, ccaihongwang78@163.com
}

\begin{abstract}
Keywords: cushion segment, forming spring back, reverse trial method, finite element simulation, experimental verification.
\end{abstract}

\begin{abstract}
Cushion segment of disc assembly for automobile clutch is taken as research object, a calculation method of forming height for cushion segment is presented based on the analysis of theory of spring back, and it is called reverse trial method. The dimension of forming die for cushion segment is obtained according to reverse trial method, finite element model of bending forming for cushion segment is established. DYNAFORM is used to simulate the spring back of cushion segment, and distribution of stress for forming spring back is analyzed. In addition, forming die is used to verify the forming height by experiment, and the experimental value is $1.55 \mathrm{~mm}$. The results show that the forming height of reverse trial method and simulation are $1.766 \mathrm{~mm}$ and $1.63 \mathrm{~mm}$ respectively, and the error between reverse trial method and simulation and experiment are $8.34 \%$ and $13.94 \%$ respectively. Therefore, the correctness of reverse trial method is verified, and it better guides the die design and production of enterprises of cushion segment.
\end{abstract}

\section{Introduction}

Cushion segment is an important elastic part in disc assembly for automobile clutch, the forming height of cushion segment has a direct influence on the axial compression performance and assembly size of disc assembly [1,2]. Cushion segment is often made by high strength spring steel of $65 \mathrm{Mn}$ or $\mathrm{C} 75 \mathrm{~S}$, and it is easy to cause spring back because of the larger ratio of yield strength and elastic modulus of material. The profile dimension of forming die is usually designed by practical experience of engineer. Therefore, it lacks a certain theoretical basis and it often needs to repair the die by $2 \sim 3$ times so that the qualified product can be obtained $[3,4,5,6]$. A theoretical calculation method for forming height of cushion segment is put forward based on the basic theory of spring back of sheet metal, and it is called reverse trial method. DYNAFORM is used to simulate the spring back of cushion segment, the theoretical calculation value of reverse trial method is compared with the finite element simulation value and the experimental value, and it can better guide the design and actual production of forming die for cushion segment.

\section{Theoretical analysis of spring back for cushion segment}

Fig. 1 is a part drawing of cushion segment. According to the basic theory of bending spring back of sheet metal, a method of calculating forming height for cushion segment is put forward in this paper, that is to say, the reverse trial method. The method of calculation is summarized as follows: Firstly, the fillet radius of cushion segment is calculated by fillet radius and depth of die based on the basic theory of spring back of sheet metal; Secondly, the bending forming height of cushion segment is calculated according to the principle of equal line length after spring back of sheet metal and the mathematical geometry relationship; When the theoretical calculation value of reverse trial method does not meet the design requirements of the drawing, the radius and depth of the die should be reset to be tested until the theoretical value is in line with the design value of the drawing. 


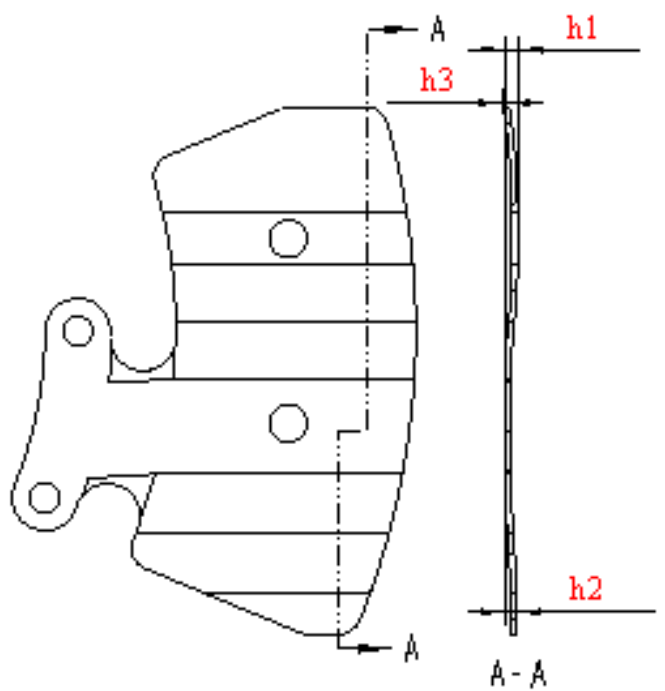

Fig. 1 Part drawing of cushion segment for clutch

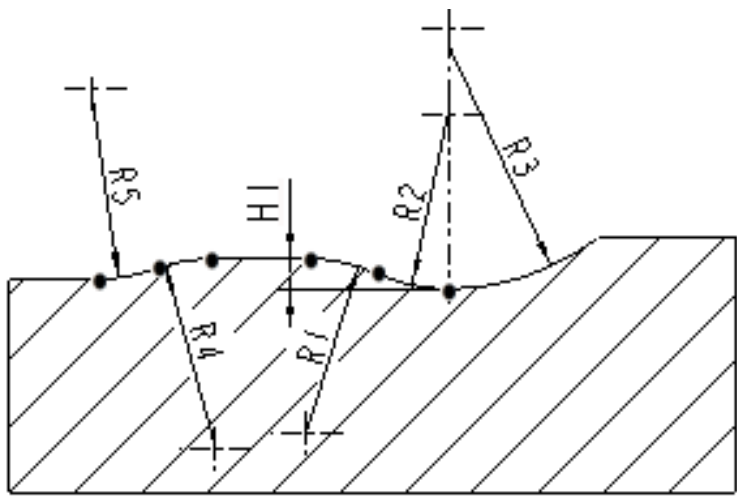

Fig. 2 Diagram of forming die for cushion segment.

Fig. 2 is a schematic diagram of bending die for cushion segment, taking the forming die as an example, the bending forming height of cushion segment (as shown in figure.1) is calculated. According to the practical experience of engineer, it is assumed that the fillet radius $R_{1}$ and $R_{2}$ of forming die for cushion segment is $23.59 \mathrm{~mm}$ and $25.5 \mathrm{~mm}$ respectively, and the depth $H_{1}$ of forming die is $2.5 \mathrm{~mm}$.

(1) According to the computational formula of spring back, the corresponding arc radius of $R_{1}$ and $R_{2}$ for $r_{1}$ and $r_{2}$ can be obtained, and its value are $57.556 \mathrm{~mm}$ and $70.427 \mathrm{~mm}$ respectively.

(2) It is deduced that the central angle of forming die is $18.364^{\circ}$ according to the computational formula of $\cos \beta=\left(R_{1}+R_{2}-H_{1}\right) /\left(R_{1}+R_{2}\right)$.

(3) The corresponding arc length of $R_{1}$ and $R_{2}$ are $\widetilde{B_{1}} \cdot \widetilde{B_{2}}$.

$\widehat{B_{1}}=\pi \cdot 2 r_{1} \times \beta / 360=7.557 \mathrm{~mm}$

$\widehat{B_{2}}=\pi \cdot 2 r_{2} \cdot \beta / 360=8.169 \mathrm{~mm}$

(4) In accordance with the principle of equal length of circle arc before and after spring back, the center angle of arc for cushion segment is calculated.

$\alpha=\left[\left(R_{1}+R_{2}\right) \cdot \beta\right] /\left(r_{1}+r_{2}\right)=7.044^{\circ}$

(5) The bending forming height of cushion segment is calculated according to the geometric relation.

$h_{1}=r_{1}+r_{2}-\left(r_{1}+r_{2}\right) \cos \alpha+t=1.766 \mathrm{~mm}$

In formula (4), $t$ represents the thickness of cushion segment.

It is derived that the theoretical calculation value of the forming height for cushion segment is 1.77 $\mathrm{mm}$, which meets the design requirements of the drawing $(1.6 \sim 1.8 \mathrm{~mm})$. In accordance with the above calculation procedure, $h_{2}$ and $h_{3}$ are calculated for $1.601 \mathrm{~mm}$ and $0.13 \mathrm{~mm}$ respectively, and it is corresponding to the die radius of $R_{4}, R_{5}$ and $R_{3}$ are $24.7 \mathrm{~mm}, 26.2 \mathrm{~mm}$ and $22.6 \mathrm{~mm}$ respectively.

\section{Finite element simulation of spring back for cushion segment}

\subsection{Establishment of finite element model.}

The radius of forming die for cushion segment is obtained based on the theoretical calculation of reverse trial method, the geometric model of die, cushion segment and punch are established by using CREO software, and it is imported into the finite element software of DYNAFORM. The finite element model of bending forming for cushion segment is obtained (as shown in figure.3). In the simulation process of spring back for cushion segment, the material of cushion segment is $65 \mathrm{Mn}$ high 
strength spring steel, the thickness of material is $0.8 \mathrm{~mm}$, the die clearance is $1.0 t$ ( $t$ is the thickness of sheet metal), the friction coefficient is 0.125 , and the virtual stamping speed is $2500 \mathrm{~mm} \cdot \mathrm{s}^{-1}$. The cushion segment is arranged as an elastic plastic body, while the punch and the die are all provided with rigid bodies. In addition, the unit formula is set as a full integral unit, and three constraint points are selected on cushion segment to limit the movement of the sheet metal.

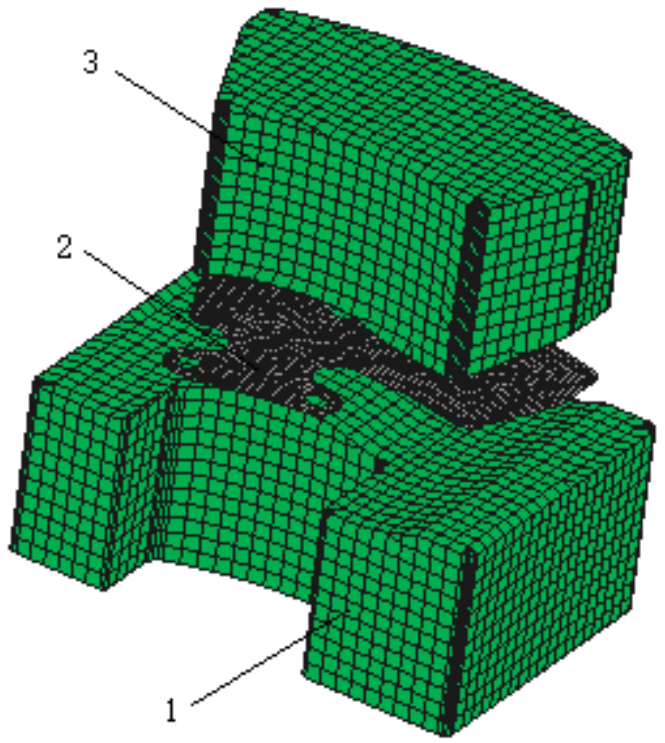

Fig. 3 Finite model of bend forming for cushion segment

1-die 2- cushion segment 3-punch

\subsection{Analysis of finite element simulation results.}

As can be seen from fig.4, the deformation of the fillet region is very severe after the forming of cushion segment. Therefore, the value of stress in the deformation region is larger. In addition, the maximum stress value of fillet for cushion segment is $1140.36 \mathrm{MPa}$ before spring back. The residual stress in the fillet region is released after spring back, which results in a reduction of the maximum stress value in the fillet region to $517.43 \mathrm{MPa}$. This is an important reason for the spring back of cushion segment, and it affects the forming height of cushion segment.
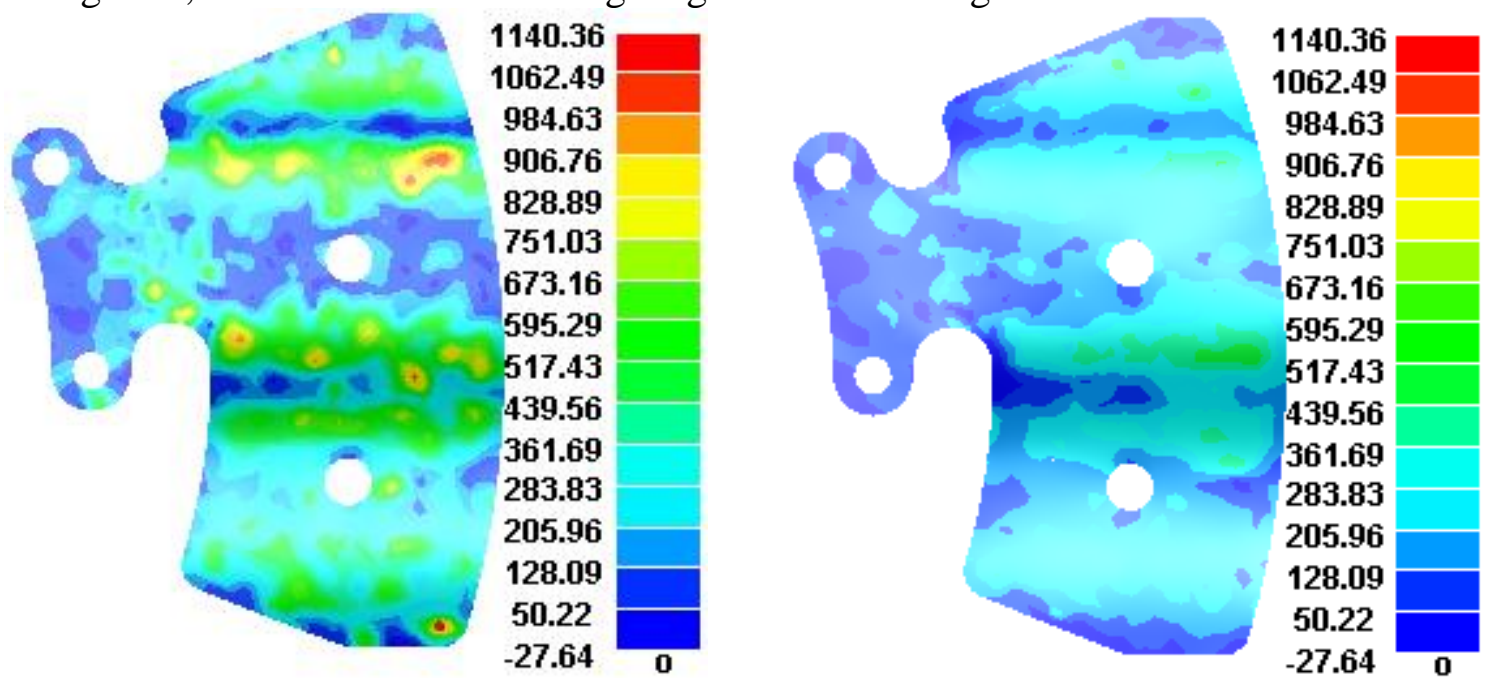

Fig.4 Comparison of stress for cushion segment at before and after spring back

(a) before spring back

(b) after spring back

The section cutting function in the post-processing of DYNAFORM software is used to cut cushion segment after spring back, and the simulation value of forming height is obtained (as shown in figure.5). As can be seen from figure.5, the forming height after spring back is $1.63 \mathrm{~mm}$.

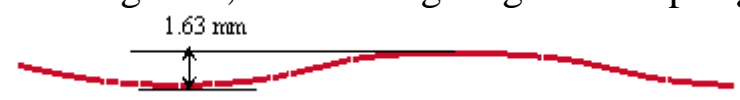

Fig. 5 Simulation results of cushion segment. 


\section{Experimental verification of spring back for cushion segment}

The reverse trial method of forming height and the correctness of finite element simulation for cushion segment are influenced by the comprehensive factors, such as the mathematical model and the establishment of boundary conditions. Therefore, H1F80 servo press and forming die of cushion segment is used to verify the correctness of reverse trial method and simulation (as shown in figure.6). The technical parameters of H1F80 servo press are: nominal pressure is $800 \mathrm{KN}$, standard stroke is 75 110 times / min, and slider travel is 100 130 mm.

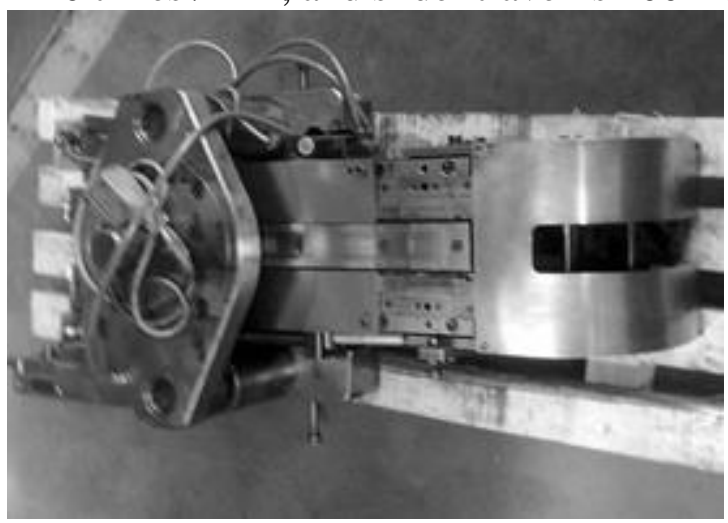

Fig. 6 Forming die of cushion segment for clutch

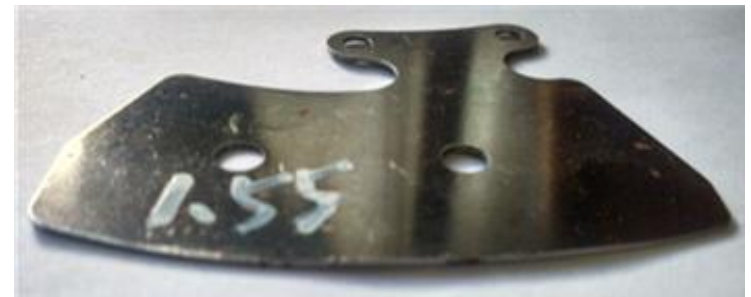

Fig. 7 Test chart of cushion segment

In the test of forming spring back for cushion segment, the material of cushion segment is $65 \mathrm{Mn}$ high strength spring steel, the thickness of material is $0.8 \mathrm{~mm}$, and the die clearance is $1.0 t$ ( $t$ is the thickness of sheet metal). In addition, digimatic height gage is used to measure the forming height of cushion segment after spring back, and the result is shown in figure.7.

As can be seen from figure.7, the experimental value of the forming height after spring back for cushion segment is $1.55 \mathrm{~mm}$, while the theoretical calculation value of reverse trial method and the finite element simulation value are $1.766 \mathrm{~mm}$ and $1.63 \mathrm{~mm}$ respectively, so the relative error between theoretical calculation value of reverse trial method and finite element simulation value and experimental value is $8.34 \%$ and $13.94 \%$ respectively. The causes of error is analyzed, and the reason is that reverse trial method and the finite element simulation of spring back for cushion segment is the ideal situation. However, the actual stamping process of forming spring back for cushion segment is affected by the material properties, machine tool and other factors, which makes the experimental value lower than the theoretical calculation value of reverse trial method and finite element simulation value. It is found that the error between the reverse trial method and the experimental value is $13.94 \%$ based on the above analysis, and the error is within the $15 \%$ acceptable error range to the actual production of the enterprise. Therefore, the validity of reverse trial method applied to predict the forming height of cushion segment is verified, which provides a train of thought for predicting the forming height of cushion segment, and it has an important guiding significance on the design and modification of the forming die for cushion segment.

\section{Summary}

A theoretical calculation method for predicting the forming height of cushion segment is presented in this paper based on the basic theory of forming spring back in sheet metal, and it is called the reverse trial method. The theoretical value of reverse trial method is simulated by using DYNAFORM finite element software, and it is found that the release of residual stress in the fillet region is the main reason for the spring back of sheet metal. Forming die of cushion segment is used to carry out the experiment, it is found that the theoretical calculation value of reverse trial method, the finite element simulation value and the experimental value of the forming height for cushion segment are $1.766 \mathrm{~mm}$, $1.63 \mathrm{~mm}$ and $1.55 \mathrm{~mm}$ respectively. The error between the theoretical calculation value and the experimental value of the reverse trial method is $13.94 \%$, which meets application requirements of actual production for enterprises. It explains the correctness of reverse trial method, and it provides a method of theoretical calculation for forming height of cushion segment. The application of reverse 
trial method can reduce die-repairing of cushion segment by 1 2 times. Therefore, it saves production costs of enterprise and improves the competitiveness of products.

\section{References}

[1]. Liu Kuiwu, Bian Wei. Study on spring back in cushion segment forming based on Dynaform [J]. Forging \& Stamping Technology. Vol. 40 (2015) No. 3, p. 1127-130.

[2]. Zhang Chi, Hu Yingcun, Liang Baoqian, etal. Study on Simulation of Spring back and Optimization Design of Die for Cushion Segment of Automobile Clutch [J]. Journal of Chongqing University of Technology (Natural Science). Vol. 26 (2012), No. 1, p. 17-21.

[3]. Yang Lijun, Dang xin,an, Ren Wei. Research on the Optimization of Technological Parameters of Sheet Metal Drawing by Orthogonal Experiment [J]. Manufacturing Technology \& Machine Tool. Vol. 10 (2006) No. 5, p. 26-29.

[4]. Huang Jinxiang. Study on Spring Back Characteristic of Indoor-sill Part Based on Disaffirm [J]. Hot Working Technology. Vol. 09 (2011), p. 176-179.

[5]. Huang Wei, Li Mengqun, Yang Yawei. Numerical analysis of spring back for V-shaped pieces based on Dynaform [J]. Forging \& Stamping Technology. Vol. 39 (2014), No. 6, p. 118-121.

[6]. Yang Taide. The pressing technology and die design of cold-forming profile [J]. Manufacturing Technology \& Machine Tool. Vol. 05 (2014), p. 34-36. 